\title{
新型含环丙基的间二酰胺类化合物的合成及其杀虫活性
}

\author{
罗春艳 ${ }^{a}$ 马文静 $b$ 吕亮 ${ }^{b}$ 庞怀林 ${ }^{b}$ 相君成 ${ }^{c}$ \\ 周丽琪 $c$ 尹笃林*,a 刘吉永*,b \\ ( ${ }^{a}$ 湖南师范大学化学化工学院 石化新材料与资源精细利用国家地方联合工程实验室＼cjkstart长沙 410081) \\ $\left({ }^{b}\right.$ 上海泰禾国际贸易有限公司 上海 200335) \\ ( ${ }^{c}$ 上海晓明检测技术服务有限公司 上海 200335)
}

\begin{abstract}
摘要 为了发现结构新颖的高效杀虫剂, 利用活性亚结构拼接的方法, 设计并合成了 11 个未见文献报道的新型含环丙 基的间二酰胺类化合物，其结构经过 ${ }^{1} \mathrm{H} \mathrm{NMR},{ }^{13} \mathrm{C} N \mathrm{NR}$ 和 HRMS 确证. 初步的生物活性测试结果表明, 部分化合物对 小菜蛾和二化蛽表现出了较好的杀虫活性. 其中 $N$-(2-溴-4-(全氟丙烷-2-基)-6-(三氟甲基)苯基)-3-( $N$-(环丙基甲基)苯甲 酰胺基)-2-氟苯甲酰胺(6d)和 3-( $N$-(环丙基甲基)苯甲酰胺基)- $N$-(2,6-二溴-4-(全氟丙烷-2-基)苯基)-2-氟苯甲酰胺(6e)在 1 $\mathrm{mg} / \mathrm{L}$ 测试浓度下对小菜蛾的杀虫活性都为 $100 \%$, 化合物 $6 \mathbf{d}$ 在 $5 \mathrm{mg} / \mathrm{L}$ 测试浓度下对二化螟的杀虫活性为 $100 \%$.

关键词 合成; 环丙基; 间二酰胺; 杀虫活性
\end{abstract}

\section{Synthesis and Insecticidal Activity of Novel meta-Diamide Compounds Containing Cyclopropyl Group}

\author{
Luo, Chunyan ${ }^{a}$ \\ Ma, Wenjing ${ }^{b}$ \\ Lv, Liang ${ }^{b}$ \\ Pang, Huailin ${ }^{b}$ \\ Zhou, Liqi ${ }^{c}$ \\ Yin, Dulin*,a \\ Liu, Jiyong ${ }^{*, b}$ \\ Xiang, Juncheng ${ }^{c}$ \\ $\left({ }^{a}\right.$ National \& Local Joint Engineering Laboratory for New Petro-chemical Materials and Fine Utilization of Resources, \\ College of Chemistry and Chemical Engineering, Hunan Normal University, Changsha 410081) \\ ( ${ }^{b}$ CAC Shanghai International Trading Co., Ltd., Shanghai 200335) \\ ( ${ }^{c}$ Greentech Laboratory Co., Ltd., Shanghai 200335)
}

\begin{abstract}
To discover novel insecticide with improved activity, a series of meta-diamide compounds containing cyclopropyl moiety were synthesized on the principle of biologically active factor splicing. The structures of synthetic derivatives were characterized by ${ }^{1} \mathrm{H}$ NMR, ${ }^{13} \mathrm{C}$ NMR and HRMS. Preliminary bioassay data showed that some of the title compounds exhibited good insecticidal activities against Plutella xylostella and Chilo suppressalis. Among them, the mortality of $N$-(2-bromo-4(perfluoropropan-2-yl)-6-(trifluoromethyl)phenyl)-3-( $N$-(cyclopropylmethyl)benzamido)-2-fluorobenzamide (6d) and 3 -( $N$ (cyclopropylmethyl)benzamido)- $N$-(2,6-dibromo-4-(perfluoropropan-2-yl)phenyl)-2-fluorobenzamide (6e) was $100 \%$ against Plute- lla xylostella at $1 \mathrm{mg} / \mathrm{L}$, and the mortality of compound $\mathbf{6 d}$ was $100 \%$ against Chilo suppressalis at $5 \mathrm{mg} / \mathrm{L}$.
\end{abstract}

Keywords synthesis; cyclopropyl; meta-diamide; inseticidal activity

21 世纪以来, 杀虫剂的发展进入高效、低毒、环境 友好时期. 双酰胺类杀虫剂因对鳞翅目害虫高效, 且与 其它常用杀虫药剂间无交互抗性, 同时对哺乳动物安 全, 因此被广泛使用 ${ }^{[1-4]}$. 双酰胺类杀虫剂的主要品种有 氟苯虫酰胺(Flubendiamide, 1)、氯虫苯甲酰胺(Chlorantraniliprole, 2) 和澳氭虫酰胺 (Cyantraniliprole, 3 $)^{[5-8]}$. 由 于氯虫苯甲酰胺等的大量推广使用, 其存在很大的抗性 风险, 多种害虫的田间种群已对其产生了抗药性 ${ }^{[9-13]}$.
溴虫氟苯双酰胺(Broflanilide, 4) 是一种全新的双酰 胺类杀虫剂, 由日本三井化学公司开发并于 2014 年公

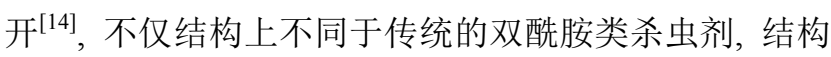
变化为间二酰胺, 而且具有新颖的作用机制, 作用于昆 虫的 $\gamma$-氨基丁酸(GABA)受体 ${ }^{[15-19]}$, 目前已经被国际杀 虫剂抗性行动委员会 (IRAC)划分为第 30 组化合物.

环丙基作为一种重要的活性基团, 是具有高度张力 的三元环状结构, 具有增强药物药效, 降低脱靶作用,

* Corresponding authors. E-mail: dulinyin@126.com and jiyong_liu@cacch.com

Received March 9, 2020; revised May 26, 2020; published online July 15, 2020. 
提高代谢稳定性, 增强药物与受体的亲和力以及改善药 物解离度等优点 ${ }^{[20-21]}$. 鉴于此, 环丙基在药物化学中被 广泛应用. 2018 年, 全球药品销售 TOP 200 的药物中有 10 种药物含有环丙基结构. 农药杀虫剂领域, 拟除虫菊 酯类化合物普遍具有环丙烷结构 ${ }^{[22]}$. 日本石原产业公 司 2013 年申请登记的环溴虫酰胺(Cyclaniliprole, 5), 同 样具有环丙基结构 ${ }^{[23]}$.<smiles>Cc1cc(C(F)(F)C(F)(F)F)ccc1NC(=O)c1cccc(I)c1C(=O)NC(C)(C)CS(C)(=O)=O</smiles><smiles>CNC(=O)c1cc(C#N)cc(C)c1NC(=O)c1cc(Br)nn1-c1ncccc1Cl</smiles>

3<smiles>CC(NC(=O)c1cc(Cl)cc(Br)c1NC(=O)c1cc(Br)nn1-c1ncccc1Cl)C1CC1</smiles>

图 1 化合物 $\mathbf{1} \sim \mathbf{5}$ 的结构式

Figure 1 Chemical structures of compounds $1 \sim 5$

为了解决抗性问题和寻找具有高杀虫活性的农药 先导化合物, 本工作采用活性亚结构拼接的方法, 以溴 虫氟苯双酰胺为先导化合物, 将环丙基结构片段引入溴 虫氟苯双酰胺中, 合成了化合物 6 , 并对其构效关系进 行了研究, 分别考察了化合物 6 中 $\mathrm{Q}^{1} 、 \mathrm{Y}^{1} 、 \mathrm{Y}^{2}$ 和 $\mathrm{Y}^{3}$ 部 分结构变化对杀虫活性的影响(Scheme 1), 以期发现具 有优良杀虫活性的化合物. 对合成的化合物进行了 ${ }^{1} \mathrm{H}$ $\mathrm{NMR},{ }^{13} \mathrm{C} \mathrm{NMR}$ 和 HRMS 表征. 初步杀虫活性测试结果 表明，部分化合物显示出了较好的杀虫活性.

\section{1 结果与讨论}

\section{1 化合物的合成}

采用 Scheme 2 所示的合成路线 ${ }^{[24-26]}$, 经 5 步反应合<smiles>[Y2]c1cc([Y2])c(NC(=O)c2cccc(N(CC3CC3)C(=O)[O-])c2F)c([Y])c1</smiles><smiles>C=C=CC(F)(F)C(F)(F)C(F)(F)F</smiles>

图示 1 目标化合物 6 的设计策略

Scheme 1 Design strategy of the title compound 6

成了目标化合物 $\mathbf{6 a} \sim \mathbf{6 k}$. 化合物的合成以 2-氟-3-氨基 苯甲酸甲酯为起始原料, 经烷基化、酰胺化和水解化反 应得到羧酸中间体 $11 \mathrm{a} \sim 11 \mathrm{k}$, 中间体 $11 \mathrm{a} \sim 11 \mathrm{k}$ 制得酰 氯与苯胺反应，最终得到目标产物 $\mathbf{6 a} \sim \mathbf{6 k}$.

\section{2 化合物的杀虫活性}

目标化合物 $\mathbf{6 a} \sim \mathbf{6 k}$ 对小菜蛾 (Plutella xylostella)和 二化螟(Chilo suppressalis)的杀虫活性测试结果见表 1 . 初步的生物活性测试结果显示, 部分目标化合物对小菜 蛾表现出较好的杀虫活性. 在测试浓度为 $1 \mathrm{mg} / \mathrm{L}$ 时, 化 合物 6d、6e、6f 对小菜蛾的杀虫活性大于或等于 $90 \%$. 当 $\mathrm{Q}^{1}$ 为苯基, $\mathrm{Y}^{1}$ 为三氟甲基， $\mathrm{Y}^{2}$ 为七氟异丙基， $\mathrm{Y}^{3}$ 为溴原 子时, 化合物 $6 \mathbf{d}$ 在测试浓度为 $1 \mathrm{mg} / \mathrm{L}$ 时对小菜蛾的死 亡率为 $100 \%$, 与对照药剂相当; 在测试浓度为 $0.1 \mathrm{mg} / \mathrm{L}$ 时对小菜蛾的死亡率为 $100 \%$ ，优于对照药剂，说明环 丙甲基的引入具有增效作用. 当 $\mathrm{Y}^{1}$ 为三氟甲基, $\mathrm{Y}^{2}$ 为七 氟异丙基, $\mathrm{Y}^{3}$ 为溴原子时, $\mathrm{Q}^{1}$ 分别选自噻吩基、异噻唑 基、吡唑基、苯基，对应化合物 $6 \mathrm{a} 、 6 \mathrm{~b} 、 6 \mathrm{c} 、 6 \mathrm{~d}$ 在测 试浓度为 $1 \mathrm{mg} / \mathrm{L}$ 时对小菜蛾的死亡率分别为 $6.67 \%$ 、 $3.33 \% 、 20.00 \% 、 100.00 \%$ ，说明 $\mathrm{Q}^{1}$ 选取苯基活性优于 五元芳杂环基. 当 $\mathrm{Q}^{1}$ 为苯基, $\mathrm{Y}^{2}$ 为七氟异丙基, $\mathrm{Y}^{3}$ 为溴 原子时， $\mathrm{Y}^{1}$ 分别选自三氟甲基、溴原子、甲基，对应化 合物 6d、6e、6f 在测试浓度为 $0.1 \mathrm{mg} / \mathrm{L}$ 时对小菜蛾的 死亡率分别为 $100.00 \% 、 20.00 \% 、 10.00 \%$, 说明在此取 代基策略下, $\mathrm{Y}^{1}$ 选取吸电性更强的三氟甲基具有增效作 用. 当 $\mathrm{Q}^{1}$ 为苯基, $\mathrm{Y}^{1}$ 为三氟甲基, $\mathrm{Y}^{3}$ 为溴原子时, $\mathrm{Y}^{2}$ 分别 选自七氟异丙基、三氟甲基，对应化合物 $6 \mathbf{d} 、 6 \mathbf{i}$ 在测试 浓度为 $1 \mathrm{mg} / \mathrm{L}$ 时对小菜蛾的死亡率分别为 $100.00 \%$ 、 $6.67 \%$, 说明 $\mathrm{Y}^{2}$ 选取亲脂性更强的七氟异丙基具有增效 作用. 对比化合物 $6 \mathrm{e}$ 和 $6 \mathbf{j}, 1 \mathrm{mg} / \mathrm{L}$ 测试浓度下对小菜蛾 
<smiles>COC(=O)c1cccc(NCC2CC2C2CC2)c1F</smiles>

$10 \mathrm{a} \sim 10 \mathrm{k}$<smiles>O=C(Cl)c1cccc(N(CC2CC2)C(=O)[O-])c1F</smiles><smiles>[Y]c1cc([Y])c(N)c(C)c1</smiles><smiles>[Y2]c1cc([Y])c(NC(=O)c2cccc(N(CC3CC3)C(=O)[O-])c2F)c([Y])c1</smiles>

6a $\sim$ 6d: $Y^{1}=\mathrm{CF}_{3}, Y^{2}={ }_{{ }_{3} \mathrm{C}_{2}}^{\mathrm{CF}_{3}} \mathrm{CF}_{3}, \mathrm{Y}^{3}=\mathrm{Br} ; \mathbf{6 e}: \mathrm{Y}^{1}=\mathrm{Br}$, $\mathrm{Y}^{2}={ }_{y_{2} \mathrm{~K}_{2}}^{\mathrm{F}_{3} \mathrm{C}} \mathrm{CF}_{\mathrm{CF}_{3}}, \mathrm{Y}^{3}=\mathrm{Br} ; 6 \mathbf{6 f}: \mathrm{Y}^{1}=\mathrm{Me}, \mathrm{Y}^{2}={ }_{3_{2}}^{\mathrm{F}_{3} \mathrm{C}} \mathrm{CF}_{3}, \mathrm{Y}^{3}=\mathrm{Br}$;

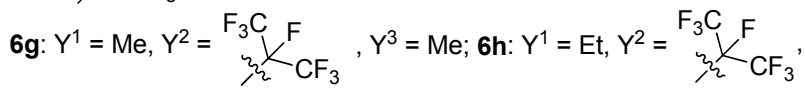
$\mathrm{Y}^{3}=\mathrm{Et} ; \mathbf{6 i}: \mathrm{Y}^{1}=\mathrm{CF}_{3}, \mathrm{Y}^{2}=\mathrm{CF}_{3}, \mathrm{Y}^{3}=\mathrm{Br} ; \mathbf{6 j}: \mathrm{Y}^{1}=\mathrm{Br}, \mathrm{Y}^{2}=\mathrm{CF}_{3} \mathrm{O}$,

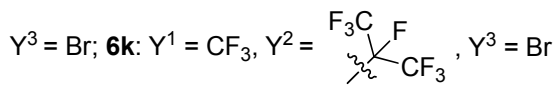

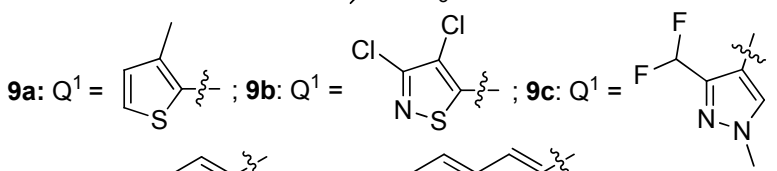
$9 \mathrm{~d} \sim 9 \mathrm{j}: \mathrm{Q}^{1}=\mathrm{W}^{\frac{\xi}{2}} ; 9 \mathrm{k}: \mathrm{Q}^{1}=$ Reagents and conditions: (i) $\mathrm{K}_{2} \mathrm{CO}_{3}$, DMF, reflux; (ii) Py., THF, r.t. or $\mathrm{Et}_{3} \mathrm{~N}$, THF, $80^{\circ} \mathrm{C}$; (iii) $\mathrm{MeOH}$, r.t.; (iv) toluene, reflux; (v) LDA, $-70^{\circ} \mathrm{C} \sim$ r.t.

图示 2 目标化合物 $6 \mathrm{a} \sim 6 \mathrm{k}$ 的合成路线

Scheme 2 Synthetic route of compounds $6 \mathbf{a} \sim \mathbf{6 k}$

的杀虫活性分别为 $100.00 \% 、 0.00 \%$, 同样说明 $\mathrm{Y}^{2}$ 选取 亲脂性更强的七氟异丙基具有增效作用. 当 $\mathrm{Q}^{1}$ 为苯基, $\mathrm{Y}^{1}$ 为甲基, $\mathrm{Y}^{2}$ 为七氟异丙基时, $\mathrm{Y}^{3}$ 分别选自溴原子、甲 基, 对应化合物 6f、6g 在测试浓度为 $1 \mathrm{mg} / \mathrm{L}$ 时对小菜 蛾的死亡率分别为 $90.00 \% 、 46.67 \%$, 说明 $\mathrm{Y}^{3}$ 选取吸电 性更强一些的溴原子活性优于甲基取代. 当 $\mathrm{Q}^{1}$ 为苯基, $\mathrm{Y}^{2}$ 为七氟异丙基, $\mathrm{Y}^{1}$ 和 $\mathrm{Y}^{3}$ 由二甲基取代变化为二乙基 取代时, 对应化合物 $6 \mathrm{~g} 、 6 \mathrm{~h}$ 在测试浓度为 $1 \mathrm{mg} / \mathrm{L}$ 时对
小菜蛾的死亡率分别为 $46.67 \% 、 83.33 \%$, 说明在此取代 基策略下，延长碳链长度具有增效作用. 对比化合物 $6 \mathbf{d}$ 和 6k 在 $1 \mathrm{mg} / \mathrm{L}$ 测试浓度下对小菜蛾的杀虫活性分别为 $100.00 \%$ 、 $10.00 \%$, 说明苯环与酰胺基团之间插入双键 化合物活性下降. 此外, 部分目标化合物对二化螟均表 现出较好的杀虫活性. 在测试浓度为 $5 \mathrm{mg} / \mathrm{L}$ 时, 化合物 6d 对二化蛽的杀虫活性为 $100.00 \%$ ，与对照药剂相当; 在测试浓度为 $1 \mathrm{mg} / \mathrm{L}$ 时二化螟的死亡率为 $80.00 \%$, 优 于对照药剂, 说明环丙甲基的引入具有增效作用. 针对 $\mathrm{Q}^{1}$ 部分、 $\mathrm{Y}^{1}$ 部分、 $\mathrm{Y}^{2}$ 部分、 $\mathrm{Y}^{3}$ 部分以及引入双键的变 化, 二化蚯与小菜蛾有类似的构效关系. 以溴虫氟苯双 酰胺为对照药剂, 对化合物 $\mathbf{6 d}$ 进行了小菜蛾和二化螟 的 $\mathrm{LD}_{50}$ 值测定，结果见表 2 和表 3 . 由表 2 和表 3 可知, 化合物 $6 \mathbf{d}$ 对小菜蛾和二化螟的 $\mathrm{LD}_{50}$ 值分别为 0.03 和 $0.64 \mathrm{mg} / \mathrm{L}$, 均优于对照药剂溴虫氟苯双酰胺. 这为今后 间二酰胺类衍生物的分子设计与生物活性测试研究提 供了重要的实验数据与结构选择模式.

表 1 目标化合物 $6 \mathbf{a} \sim 6 \mathbf{k}$ 的杀虫活性(死亡率/\%) ${ }^{a}$ Table 1 Insecticidal activities of title compounds $6 \mathbf{a} \sim 6 \mathbf{6}$

\begin{tabular}{|c|c|c|c|c|c|}
\hline \multirow{2}{*}{ Compd. } & \multicolumn{2}{|c|}{ Plutella xylostella } & \multicolumn{3}{|c|}{ Chilo suppressalis } \\
\hline & $1 \mathrm{mg} / \mathrm{L}$ & $0.1 \mathrm{mg} / \mathrm{L}$ & $10 \mathrm{mg} / \mathrm{L}$ & $5 \mathrm{mg} / \mathrm{L}$ & $1 \mathrm{mg} / \mathrm{L}$ \\
\hline $6 a$ & 6.67 & - & 0.00 & - & - \\
\hline $6 \mathbf{b}$ & 3.33 & - & 3.33 & - & - \\
\hline $6 c$ & 20.00 & - & 13.33 & - & - \\
\hline 6d & 100.00 & 100.00 & 100.00 & 100.00 & 80.00 \\
\hline $6 e$ & 100.00 & 20.00 & - & 16.67 & - \\
\hline $6 f$ & 90.00 & 10.00 & 一 & 0.00 & - \\
\hline $6 \mathrm{~g}$ & 46.67 & - & - & 0.00 & - \\
\hline $6 h$ & 83.33 & - & 一 & 40.00 & - \\
\hline $6 \mathbf{i}$ & 6.67 & - & - & 0.00 & - \\
\hline $6 \mathbf{j}$ & 0.00 & 一 & 一 & 0.00 & - \\
\hline $6 \mathbf{k}$ & 10.00 & - & - & 0.00 & - \\
\hline $\begin{array}{c}\text { 溴虫氟苯 } \\
\text { 双酰胺 }\end{array}$ & 100.00 & 66.67 & 100.00 & 100.00 & 33.33 \\
\hline
\end{tabular}

表 2 目标化合物 $6 \mathbf{d}$ 对小菜蛾的 $\mathrm{LD}_{50}$ 值

Table $2 \mathrm{LD}_{50}$ value of compound $\mathbf{6 d}$ against Plutella xylostella

\begin{tabular}{ccccc}
\hline 药剂 & $\begin{array}{c}\mathrm{LD}_{50} / \\
\left(\mathrm{mg} \cdot \mathrm{L}^{-1}\right)\end{array}$ & 线性回归方程 & \multicolumn{2}{c}{ 相关系数 标准误差 } \\
\hline $\begin{array}{c}\mathbf{6 d} \\
0.03\end{array}$ & $y=8.11 x+17.41$ & 0.94 & 0.02 \\
$\begin{array}{c}\text { 溴虫氟苯 } \\
\text { 双酰胺 }\end{array}$ & 0.07 & $y=9.51 x+16.08$ & 0.95 & 0.03 \\
\hline
\end{tabular}

表 3 目标化合物 $\mathbf{6 d}$ 对二化蛽的 $\mathrm{LD}_{50}$ 值

Table $3 \quad \mathrm{LD}_{50}$ value of compound $\mathbf{6 d}$ against Chilo suppressalis

\begin{tabular}{ccccc}
\hline 药剂 & $\begin{array}{c}\mathrm{LD}_{50} / \\
\left(\mathrm{mg} \cdot \mathrm{L}^{-1}\right)\end{array}$ & 线性回归方程 & \multicolumn{2}{c}{ 相关系数 标准误差 } \\
\hline $\mathbf{6 d}$ & 0.64 & $y=9.18 x+6.77$ & 0.96 & 0.42 \\
$\begin{array}{c}\text { 溴虫氟苯 } \\
\text { 双酰胺 }\end{array}$ & 1.27 & $y=7.91 x+4.17$ & 0.98 & 0.88 \\
\hline
\end{tabular}




\section{2 结论}

本工作利用活性亚结构拼接的方法, 设计并合成了 11 个新型含环丙基的间二酰胺类化合物, 并通过 ${ }^{1} \mathrm{H}$ $\mathrm{NMR},{ }^{13} \mathrm{C} \mathrm{NMR}$ 和 HRMS 对目标化合物进行了结构表 征. 杀虫活性测试表明, 部分化合物对小菜蛾和二化螟 表现出了较好的杀虫活性. 其中化合物 6d、6e 在 $1 \mathrm{mg} / \mathrm{L}$ 测试浓度下对小菜蛾的杀虫活性为 $100 \%$, 化合物 $6 \mathbf{d}$ 在 $5 \mathrm{mg} / \mathrm{L}$ 测试浓度下对二化螟的杀虫活性为 $100 \%$. 化合 物 6d 对小菜蛾和二化螟都表现出了优异的杀虫活性, $\mathrm{LD}_{50}$ 值分别为 0.03 和 $0.64 \mathrm{mg} / \mathrm{L}$, 均优于对照药剂溴虫 氟苯双酰胺, 具有一定的应用价值. 目前该类化合物的 结构衍生与生物活性研究正在进行中.

\section{3 实验部分}

\section{1 仪器与试剂}

Mercury 400 (Varian)或 Mercury 500 (Varian)型核磁 共振仪, TMS 为内标, DMSO- $d_{6}$ 或 $\mathrm{CDCl}_{3}$ 为溶剂; Agilent 1290-6230 TOF 高分辨质谱仪(HRMS); Agilent 12606120 Quadrupole 液相 - 质谱仪 (LRMS); 层析硅胶 (GF245)薄板(涤纶片基, $5 \times 10 \mathrm{~cm}$ ); 快速柱层析用硅胶 (ZCX-II, 粗孔 100 140 目)为青岛海洋化工厂产品. 所 用溶剂和试剂均为市售, 分析纯或化学纯, 必要时经过 干燥或蒸馏处理.

\section{2 中间体及目标化合物的合成}

\subsection{1中间体 $\mathbf{1 0}$ 的合成}

向装有磁子的反应瓶中依次加 2-氟-3-胺基苯甲酸 甲酯( $20 \mathrm{~g}, 118.23 \mathrm{mmol}) 、$ 溴甲基环丙烷(20.75 g, 153.70 $\mathrm{mmol}) 、$ 碳酸钾 $(21.24 \mathrm{~g}, 153.70 \mathrm{mmol}) 、 N, N$-二甲基甲酰 胺 $(200 \mathrm{~mL})$, 在回流条件下搅拌 $16 \mathrm{~h}$, 层色谱(TLC)监测 至反应不再进行时, 关闭加热, 结束反应. 待反应液冷 至室温后, 向反应液中加入水 $(200 \mathrm{~mL})$, 用乙酸乙酯 $(100 \mathrm{~mL})$ 萃取, 有机层经饱和食盐水洗涤、无水硫酸钠 干燥后, 减压下浓缩, 残余物经柱色谱提纯 [淋洗液为 $V($ 石油醚 $): V($ 乙酸乙酯 $)=10 ： 1]$ 得 $13.00 \mathrm{~g}$ 淡黄色液体 产物 2-氟-3-[N-(环丙甲基)胺基]苯甲酸甲酯 $(\mathbf{8})$, 产率 49.39\%. ${ }^{1} \mathrm{H}$ NMR (400 MHz, $\mathrm{CDCl}_{3}$ ) $\delta: 7.15$ (t, $J=7.7 \mathrm{~Hz}$, $1 \mathrm{H}), 7.01$ (t, $J=7.9 \mathrm{~Hz}, 1 \mathrm{H}), 6.82$ (t, $J=8.4 \mathrm{~Hz}, 1 \mathrm{H}), 4.18$ (s, 1H), $3.91(\mathrm{~s}, 3 \mathrm{H}), 3.00(\mathrm{~d}, J=6.9 \mathrm{~Hz}, 2 \mathrm{H}), 1.17 \sim 1.07$ $(\mathrm{m}, 1 \mathrm{H}), 0.58$ (q, $J=5.5 \mathrm{~Hz}, 2 \mathrm{H}), 0.26$ (q, $J=4.9 \mathrm{~Hz}, 2 \mathrm{H})$; ${ }^{13} \mathrm{C}$ NMR $\left(100 \mathrm{MHz}, \mathrm{CDCl}_{3}\right) \delta: 137.8,137.7,124.0,123.9$, 117.9, 115.6, 115.6, 52.1, 48.6, 10.6, 3.5. HRMS (ESI) cacld for $\mathrm{C}_{12} \mathrm{H}_{15} \mathrm{FNO}_{2}[\mathrm{M}+\mathrm{H}]^{+}$224.1087, found 224.1082 .

将 8 (10.00 g, $44.82 \mathrm{mmol})$ 溶解在四氢呋喃 $(100 \mathrm{~mL})$
中, 加入吡啶(4.25 g, $53.78 \mathrm{mmol}$ ), 滴加 3-甲基噻吩-2甲酰氯(9a) (8.60 g, $53.78 \mathrm{mmol})$, 常温摚拌 4 h. TLC 监 测至反应不再进行时, 结束反应. 向反应液中加入乙酸 乙酯 $(50 \mathrm{~mL})$, 有机层经依次用 $2 \mathrm{~mol} \cdot \mathrm{L}^{-1}$ 盐酸溶液和饱 和碳酸氢钠溶液洗涤，经无水硫酸钠干燥后，减压下浓 缩, 残余物经柱色谱提纯 [淋洗液为 $V$ (石油醚) : $V$ (乙酸 乙酯) $=8: 1$ ], 得 $13.00 \mathrm{~g}$ 无色液体产物 2-氟-3-[ $N$-(环丙 甲基)-3-甲基噻吩-2-甲酰胺基]苯甲酸甲酯(10a), 产率 83.54\%. ${ }^{1} \mathrm{H}$ NMR (400 MHz, $\left.\mathrm{CDCl}_{3}\right) \delta: 7.85$ (t, $J=7.9 \mathrm{~Hz}$, 1H), 7.39 (t, $J=7.2 \mathrm{~Hz}, 1 \mathrm{H}), 7.13$ (t, $J=7.9 \mathrm{~Hz}, 1 \mathrm{H}), 7.08$ $(\mathrm{d}, J=4.9 \mathrm{~Hz}, 1 \mathrm{H}), 6.68(\mathrm{~d}, J=4.9 \mathrm{~Hz}, 1 \mathrm{H}), 3.91(\mathrm{~s}, 3 \mathrm{H})$, $3.71(\mathrm{~s}, 2 \mathrm{H}), 2.35$ (s, 3H), $1.08 \sim 0.98(\mathrm{~m}, 1 \mathrm{H}), 0.48 \sim 0.36$ $(\mathrm{m}, 2 \mathrm{H}), 0.09$ (q, $J=5.0 \mathrm{~Hz}, 2 \mathrm{H}) ;{ }^{13} \mathrm{C}$ NMR $(100 \mathrm{MHz}$, $\left.\mathrm{CDCl}_{3}\right) \delta: 165.1,141.6,135.4,131.4,129.8,129.3,123.8$, $123.7,53.9,52.5,15.3,9.6,3.5$. HRMS (ESI) cacld for $\mathrm{C}_{18} \mathrm{H}_{19} \mathrm{FNO}_{3} \mathrm{~S}[\mathrm{M}+\mathrm{H}]^{+}$348.1087, found 348.1063.

采用与中间体 $10 \mathrm{a}$ 类似的合成方法合成了中间体 $10 \mathrm{~b} \sim 10 \mathrm{k}$.

2-氟-3-[ $N$-(环丙甲基)-3,4-二氯异噻唑-5-甲酰胺基] 苯甲酸甲酯(10b): 淡黄色液体，产率 $67.24 \%$. ${ }^{1} \mathrm{H}$ NMR $\left(400 \mathrm{MHz}, \mathrm{CDCl}_{3}\right) \delta: 7.95(\mathrm{t}, J=6.8 \mathrm{~Hz}, 1 \mathrm{H}), 7.49$ (t, $J=$ $6.8 \mathrm{~Hz}, 1 \mathrm{H}), 7.20$ (t, $J=7.7 \mathrm{~Hz}, 1 \mathrm{H}), 3.94(\mathrm{~s}, 3 \mathrm{H}), 3.89 \sim$ $3.85(\mathrm{~m}, 1 \mathrm{H}), 3.68 \sim 3.63(\mathrm{~m}, 1 \mathrm{H}), 1.02(\mathrm{~s}, 1 \mathrm{H}), 0.49(\mathrm{~d}$, $J=7.0 \mathrm{~Hz}, 2 \mathrm{H}), 0.16$ (s, 2H); ${ }^{13} \mathrm{C} \mathrm{NMR}\left(100 \mathrm{MHz}, \mathrm{CDCl}_{3}\right)$ $\delta: 163.6,159.4,159.0,148.3,134.7,133.1,124.3,124.3$, 53.9, 52.7, 9.2, 3.9, 3.3. HRMS (ESI) cacld for $\mathrm{C}_{16} \mathrm{H}_{14} \mathrm{Cl}_{2-}$ $\mathrm{FN}_{2} \mathrm{O}_{3} \mathrm{~S}[\mathrm{M}+\mathrm{H}]^{+}$403.0086, found 403.0083.

2-氟-3-[ $N$-(环丙甲基)-3-(二氟甲基)-1-甲基- $1 H$-吡 唑-4-甲酰胺基]苯甲酸甲酯(10c)：淡黄色液体，产率 63.53\%. ${ }^{1} \mathrm{H}$ NMR (400 MHz, $\mathrm{CDCl}_{3}$ ) $\delta: 7.96$ (t, $J=6.3 \mathrm{~Hz}$, $1 \mathrm{H}), 7.47$ (t, $J=6.8 \mathrm{~Hz}, 1 \mathrm{H}), 7.24$ (dd, $J=13.8,5.8 \mathrm{~Hz}$, 2H), $7.14(\mathrm{t}, J=52.0 \mathrm{~Hz}, 1 \mathrm{H}), 6.47(\mathrm{~s}, 1 \mathrm{H}), 3.95(\mathrm{~s}, 3 \mathrm{H})$, $3.84(\mathrm{~s}, 1 \mathrm{H}), 3.71(\mathrm{~s}, 3 \mathrm{H}), 3.55(\mathrm{~s}, 1 \mathrm{H}), 1.04 \sim 0.98(\mathrm{~m}, 1 \mathrm{H})$, $0.44(\mathrm{~d}, J=7.8 \mathrm{~Hz}, 2 \mathrm{H}), 0.10(\mathrm{~d}, J=4.3 \mathrm{~Hz}, 2 \mathrm{H}) ;{ }^{13} \mathrm{C}$ NMR $\left(100 \mathrm{MHz}, \mathrm{CDCl}_{3}\right) \delta: 164.0,162.5,156.4,146.9$, $135.4,132.1,130.7,124.3,124.3,120.3,120.2,115.4$, $115.4,112.1,109.8,107.4,53.6,52.6,39.4,9.3,3.8,3.2$. HRMS (ESI) cacld for $\mathrm{C}_{18} \mathrm{H}_{19} \mathrm{~F}_{3} \mathrm{~N}_{3} \mathrm{O}_{3}[\mathrm{M}+\mathrm{H}]^{+} 382.1379$, found 382.1377

2-氟-3-[ $N$-(环丙甲基) 苯甲酰胺基]苯甲酸甲酯 $(\mathbf{1 0 d} \sim \mathbf{1 0 j})$ : 淡黄色液体，产率 $70.28 \%$. ${ }^{1} \mathrm{H}$ NMR (400 $\left.\mathrm{MHz}, \mathrm{CDCl}_{3}\right) \delta: 7.78(\mathrm{t}, J=6.1 \mathrm{~Hz}, 1 \mathrm{H}), 7.37 \sim 7.14(\mathrm{~m}$, $6 \mathrm{H}), 7.09 \sim 7.01(\mathrm{~m}, 1 \mathrm{H}), 3.91(\mathrm{~s}, 4 \mathrm{H}), 3.59(\mathrm{~s}, 1 \mathrm{H}), 1.05$ $(\mathrm{s}, 1 \mathrm{H}), 0.43(\mathrm{~d}, J=7.9 \mathrm{~Hz}, 2 \mathrm{H}), 0.10(\mathrm{~s}, 2 \mathrm{H}) ;{ }^{13} \mathrm{C} \mathrm{NMR}$ $\left(100 \mathrm{MHz}, \mathrm{CDCl}_{3}\right) \delta: 164.1,135.6,135.3,131.1,129.8$, 
127.9, 127.7, 123.8, 123.8, 53.6, 52.4, 9.5, 3.8, 3.2. HRMS (ESI) cacld for $\mathrm{C}_{19} \mathrm{H}_{19} \mathrm{FNO}_{3}[\mathrm{M}+\mathrm{H}]^{+}$328.1349, found 328.1344 .

2-氟-3-[ $N$-(环丙甲基)肉桂酰胺]苯甲酸甲酯(10k): 淡黄色液体, 产率 $30.33 \% .{ }^{1} \mathrm{H}$ NMR (400 $\left.\mathrm{MHz}, \mathrm{CDCl}_{3}\right)$ $\delta: 8.01(\mathrm{t}, J=6.7 \mathrm{~Hz}, 1 \mathrm{H}), 7.77(\mathrm{~d}, J=6.3 \mathrm{~Hz}, 1 \mathrm{H}), 7.57 \sim$ $7.49(\mathrm{~m}, 2 \mathrm{H}), 7.42 \sim 7.37(\mathrm{~m}, 2 \mathrm{H}), 7.29(\mathrm{~s}, 3 \mathrm{H}), 6.47(\mathrm{~d}$, $J=16.0 \mathrm{~Hz}, 1 \mathrm{H}), 6.20(\mathrm{~d}, J=15.4 \mathrm{~Hz}, 1 \mathrm{H}), 3.96(\mathrm{~s}, 3 \mathrm{H})$, $3.95 \sim 3.89(\mathrm{~m}, 1 \mathrm{H}), 3.50(\mathrm{dd}, J=14.0,7.3 \mathrm{~Hz}, 1 \mathrm{H}), 0.98$ $(\mathrm{d}, J=7.1 \mathrm{~Hz}, 1 \mathrm{H}), 0.43(\mathrm{~d}, J=7.9 \mathrm{~Hz}, 2 \mathrm{H}), 0.11(\mathrm{t}, J=5.0$ $\mathrm{Hz}, 2 \mathrm{H}) ;{ }^{13} \mathrm{C} \mathrm{NMR}\left(100 \mathrm{MHz}, \mathrm{CDCl}_{3}\right) \delta: 171.5,146.6$, $143.4,135.6,134.9,132.1,130.6,129.8,128.9$, 128.7, $128.3,127.9,124.3,124.2,117.5,53.0,52.6,9.6,3.9,3.2$. HRMS (ESI) cacld for $\mathrm{C}_{21} \mathrm{H}_{21} \mathrm{FNO}_{3}[\mathrm{M}+\mathrm{H}]^{+} 354.1505$, found 354.1497.

\subsection{2中间体 $\mathbf{1 1}$ 的合成}

将 10a (13.00 g, $37.42 \mathrm{mmol})$ 溶解在甲醇 $(130 \mathrm{~mL})$ 中, 加入 $10 \%$ 的氢氧化钠水溶液 $(5.99 \mathrm{~g}, 149.68 \mathrm{mmol}$, $59.9 \mathrm{~mL}$ ), 常温搅拌 $2 \mathrm{~h}$ 后, TLC 监测反应完全. 减压下 浓缩除去甲醇, 将浓缩后的残渣溶解在水 $(100 \mathrm{~mL})$ 中, 用乙酸乙酯 $(50 \mathrm{~mL})$ 萃取, 舍弃有机相, 用 $2 \mathrm{~mol} \cdot \mathrm{L}^{-1}$ 盐 酸水溶液调节水相的 $\mathrm{pH}$ 为 7 , 继续用乙酸乙酯 $(100 \mathrm{~mL})$ 萃取, 有机层经饱和食盐水洗涤、无水硫酸钠干燥后, 减压下浓缩得 $10.00 \mathrm{~g}$ 白色固体产物 2-氟-3-[N-(环丙甲 基)-3-甲基噻吩-2-甲酰胺基]苯甲酸(11a), 产率 80.16\%. m.p. $128 \sim 129{ }^{\circ} \mathrm{C} ;{ }^{1} \mathrm{H}$ NMR (400 MHz, $\left.\mathrm{CDCl}_{3}\right) \delta: 7.93$ (t, $J=6.6 \mathrm{~Hz}, 1 \mathrm{H}), 7.47$ (t, $J=6.8 \mathrm{~Hz}, 1 \mathrm{H}), 7.17$ (t, $J=7.9$ $\mathrm{Hz}, 1 \mathrm{H}), 7.09$ (d, $J=4.9 \mathrm{~Hz}, 1 \mathrm{H}), 6.70$ (d, $J=4.9 \mathrm{~Hz}, 1 \mathrm{H})$, $3.74(\mathrm{~s}, 2 \mathrm{H}), 2.35(\mathrm{~s}, 3 \mathrm{H}), 1.12 \sim 1.00(\mathrm{~m}, 1 \mathrm{H}), 0.50 \sim 0.41$ $(\mathrm{m}, 2 \mathrm{H}), 0.11(\mathrm{~d}, J=15.0 \mathrm{~Hz}, 2 \mathrm{H}) ;{ }^{13} \mathrm{C}$ NMR $(100 \mathrm{MHz}$, $\left.\mathrm{CDCl}_{3}\right) \delta: 168.1,165.5,141.8,136.1,132.1,129.9,129.1$, $123.9,123.9,54.1,15.3,9.5,3.5$. HRMS (ESI) cacld for $\mathrm{C}_{17} \mathrm{H}_{15} \mathrm{FNO}_{3} \mathrm{~S}[\mathrm{M}-\mathrm{H}]^{-}$332.0757, found 332.0755.

采用与中间体 $11 \mathrm{a}$ 类似的合成方法合成了 $11 \mathrm{~b} \sim$ $11 \mathrm{k}$.

2-氟-3-[ $N$-(环丙甲基)-3,4-二氯异噻唑-5-甲酰胺基] 苯甲酸(11b): 白色固体，产率 $75.24 \%$, m.p. 132 $133{ }^{\circ} \mathrm{C} ;{ }^{1} \mathrm{H}$ NMR (400 MHz, $\left.\mathrm{CDCl}_{3}\right) \delta$ : $7.90(\mathrm{~s}, 1 \mathrm{H})$, $7.31 \sim 7.19(\mathrm{~m}, 1 \mathrm{H}), 7.05(\mathrm{t}, J=7.9 \mathrm{~Hz}, 1 \mathrm{H}), 6.89(\mathrm{t}, J=$ $7.7 \mathrm{~Hz}, 1 \mathrm{H}), 3.01(\mathrm{~d}, J=6.9 \mathrm{~Hz}, 2 \mathrm{H}), 1.13$ (tt, $J=12.4,6.2$ $\mathrm{Hz}, 1 \mathrm{H}), 0.67 \sim 0.50(\mathrm{~m}, 2 \mathrm{H}), 0.27(\mathrm{q}, J=5.0 \mathrm{~Hz}, 2 \mathrm{H}) ;{ }^{13} \mathrm{C}$ NMR (100 MHz, $\left.\mathrm{CDCl}_{3}\right) \delta: 170.3,137.9,137.7,124.1$, 118.6, 116.9, 116.8, 116.8, 116.7, 48.7, 10.6, 3.5. HRMS (ESI) cacld for $\mathrm{C}_{15} \mathrm{H}_{10} \mathrm{Cl}_{2} \mathrm{FN}_{2} \mathrm{O}_{3} \mathrm{~S}[\mathrm{M}-\mathrm{H}]^{-} 386.9773$, found 386.9769 .
2-氟-3-[ $N$-(环丙甲基)-3-(二氟甲基)-1-甲基- $1 H$-吡 唑-4-甲酰胺基]苯甲酸(11c)：白色固体，产率 73.68\%, m.p. $131 \sim 132{ }^{\circ} \mathrm{C} ;{ }^{1} \mathrm{H}$ NMR (400 MHz, $\mathrm{CDCl}_{3}$ ) $\delta: 10.16$ (s, 1H), $7.92(\mathrm{t}, J=6.6 \mathrm{~Hz}, 1 \mathrm{H}), 7.88(\mathrm{~s}, 0 \mathrm{H}), 7.42$ (t, $J=$ $6.9 \mathrm{~Hz}, 1 \mathrm{H}), 7.20 \sim 7.12(\mathrm{~m}, 2 \mathrm{H}), 7.00(\mathrm{t}, J=52.0 \mathrm{~Hz}, 1 \mathrm{H})$, $6.46(\mathrm{~s}, 1 \mathrm{H}), 3.74(\mathrm{~s}, 1 \mathrm{H}), 3.62(\mathrm{~s}, 3 \mathrm{H}), 3.49(\mathrm{~s}, 1 \mathrm{H})$, $0.96 \sim 0.83(\mathrm{~m}, 1 \mathrm{H}), 0.34$ (q, $J=5.1 \mathrm{~Hz}, 2 \mathrm{H}), 0.01(\mathrm{~d}, J=$ $4.2 \mathrm{~Hz}, 2 \mathrm{H}) ;{ }^{13} \mathrm{C}$ NMR $\left(100 \mathrm{MHz}, \mathrm{CDCl}_{3}\right) \delta: 167.1,162.9$, 136.0, 132.6, 131.0, 124.4, 115.2, 109.8, 107.4, 53.8, 39.4, 9.3, 3.8, 3.25. HRMS (ESI) cacld for $\mathrm{C}_{17} \mathrm{H}_{15} \mathrm{~F}_{3} \mathrm{~N}_{3} \mathrm{O}_{3}[\mathrm{M}-$ $\mathrm{H}]^{-}$366.1066, found 366.1058 .

2-氟-3-[ $N$-(环丙甲基)-苯甲酰胺基]苯甲酸(11d 11j): 白色固体，产率 $80.48 \%$, m.p. $107 \sim 108{ }^{\circ} \mathrm{C} ;{ }^{1} \mathrm{H}$ NMR (400 MHz, $\left.\mathrm{CDCl}_{3}\right) \delta: 10.09(\mathrm{~s}, 1 \mathrm{H}), 7.86(\mathrm{~s}, 1 \mathrm{H})$, $7.44 \sim 7.04(\mathrm{~m}, 7 \mathrm{H}), 3.94(\mathrm{~s}, 1 \mathrm{H}), 3.65(\mathrm{~s}, 1 \mathrm{H}), 1.06(\mathrm{~s}$, $1 \mathrm{H}), 0.44$ (d, $J=7.9 \mathrm{~Hz}, 2 \mathrm{H}), 0.11(\mathrm{~s}, 2 \mathrm{H}) ;{ }^{13} \mathrm{C}$ NMR $(100$ $\left.\mathrm{MHz}, \mathrm{CDCl}_{3}\right) \delta: 171.5,167.7,135.9,135.3,131.8,130.0$, 127.9, 127.7, 123.9, 123.9, 119.1, 53.8, 9.4, 3.8, 3.2. HRMS (ESI) cacld for $\mathrm{C}_{18} \mathrm{H}_{15} \mathrm{FNO}_{3}[\mathrm{M}-\mathrm{H}]^{-}$312.1036, found 312.1034 .

2-氟-3-[ $N$-(环丙甲基)-肉桂酰胺]苯甲酸甲酯(11k): 白色固体，产率 $50.22 \%$, m.p. $165 \sim 166{ }^{\circ} \mathrm{C} ;{ }^{1} \mathrm{H}$ NMR $\left(400 \mathrm{MHz} \mathrm{CDCl}_{3}\right) \delta: 8.11(\mathrm{t}, J=6.7 \mathrm{~Hz}, 1 \mathrm{H}), 7.77(\mathrm{~d}, J=$ $15.4 \mathrm{~Hz}, 1 \mathrm{H}), 7.57$ (t, $J=6.7 \mathrm{~Hz}, 1 \mathrm{H}), 7.29$ (t, $J=14.0 \mathrm{~Hz}$, $6 \mathrm{H}), 6.21(\mathrm{~d}, J=15.4 \mathrm{~Hz}, 1 \mathrm{H}), 3.95(\mathrm{dd}, J=14.0,7.1 \mathrm{~Hz}$, $1 \mathrm{H}), 3.52(\mathrm{dd}, J=14.0,7.2 \mathrm{~Hz}, 1 \mathrm{H}), 1.07 \sim 0.96(\mathrm{~m}, 1 \mathrm{H})$, $0.44(\mathrm{~d}, J=7.8 \mathrm{~Hz}, 2 \mathrm{H}), 0.12(\mathrm{t}, J=5.0 \mathrm{~Hz}, 2 \mathrm{H}) ;{ }^{13} \mathrm{C} \mathrm{NMR}$ $\left(100 \mathrm{MHz}, \mathrm{DMSO}-d_{6}\right) \delta: 142.0,135.2,131.7,129.8,128.8$, $127.8,120.7,118.0,52.3,9.5,3.4,2.9$. HRMS (ESI) cacld for $\mathrm{C}_{20} \mathrm{H}_{17} \mathrm{FNO}_{3}[\mathrm{M}-\mathrm{H}]^{-}$338.1192, found 338.1193.

\subsection{3 目标化合物 6 的合成}

向反应瓶中依次加 2-氟-3-( $N$-(环丙甲基)苯甲酰胺 基)苯甲酸(0.40 g, $1.28 \mathrm{mmol})$ 、甲苯 $(6 \mathrm{~mL})$, 二氯亚砜 (0.75 g, $6.40 \mathrm{mmol})$, 在 $140{ }^{\circ} \mathrm{C}$ 下搅拌反应 $2 \mathrm{~h}$, 减压下 浓缩, 将浓缩后的残渣溶解在四氢呋喃 $(3 \mathrm{~mL})$ 中待用. 将 2-溴-6-三氟甲基-4-七氟异丙基苯胺(0.52 g, 1.28 $\mathrm{mmol})$ 溶解在四氢呋喃 $(4 \mathrm{~mL})$ 中, 在 $-70{ }^{\circ} \mathrm{C}$ 下滴加二异 丙基氨基锂 $(0.77 \mathrm{~mL}, 1.54 \mathrm{mmol}), 5 \mathrm{~min}$ 后滴加上一步 合成的 2-氟-3-[N-(环丙甲基)苯甲酰胺基]苯甲酰氯的四 氢呋喃溶液, 在 $-70{ }^{\circ} \mathrm{C}$ 下搅拌 $30 \mathrm{~min}$, 升至室温继续 搅拌 $30 \mathrm{~min}$. TLC 监测至反应不再进行时结束反应. 向 反应液中加入水 $(20 \mathrm{~mL})$, 用乙酸乙酯 $(20 \mathrm{~mL})$ 萃取, 有 机层经饱和食盐水洗涤、无水硫酸钠干燥后，减压下浓 缩, 残余物经柱色谱提纯 [淋洗液为 $V$ (石油醚) : $V$ (乙酸 乙酯 $)=3 ： 1]$ 得 $0.25 \mathrm{~g} N$-[2-溴-4-(1,1,1,2,3,3,3-七氟丙-2- 
基)-6-(三氟甲基)苯基]-3-[ $N$-(环丙甲基)苯甲酰胺基]-2氟苯甲酰胺 $(\mathbf{6 d})$, 产率 $27.84 \%$.

采用与目标化合物 $6 \mathrm{~d}$ 类似的方法合成 $\mathbf{6 a} \sim \mathbf{6 c}$ 、

$\mathbf{6 e} \sim 6 \mathbf{k}$.

$N$-[2-溴-4-(1,1,1,2,3,3,3-七氟丙-2-基)-6-(三氟甲基) 苯基]-3-[ $N$-(环丙甲基)-3-甲基噻吩-2-甲酰胺基]-2-氟苯 甲酰胺(6a): 黄色固体, 产率 30.14\%, m.p. $159 \sim 160{ }^{\circ} \mathrm{C}$; ${ }^{1} \mathrm{H}$ NMR (400 MHz, DMSO- $d_{6}$ ) $\delta$ : $10.66(\mathrm{~s}, 1 \mathrm{H}), 8.43$ (s, 1H), $7.96(\mathrm{~s}, 1 \mathrm{H}), 7.63(\mathrm{dt}, J=15.2,6.9 \mathrm{~Hz}, 2 \mathrm{H}), 7.46(\mathrm{~d}$, $J=4.7 \mathrm{~Hz}, 1 \mathrm{H}), 7.35$ (t, $J=7.8 \mathrm{~Hz}, 1 \mathrm{H}), 6.81$ (d, $J=4.8$ $\mathrm{Hz}, 1 \mathrm{H}), 3.66$ (s, 2H), 2.25 (s, 3H), 1.03 (s, 1H), 0.42 (d, $J=7.8 \mathrm{~Hz}, 2 \mathrm{H}), 0.15 \sim 0.05(\mathrm{~m}, 2 \mathrm{H}) ;{ }^{13} \mathrm{C}$ NMR $(100 \mathrm{MHz}$, $\left.\mathrm{CDCl}_{3}\right) \delta: 165.2,161.0,156.8\left(\mathrm{~d},{ }^{1} J_{\mathrm{C}-\mathrm{F}}=248.1 \mathrm{~Hz}\right), 141.5$, 137.3, 136.7, $135.9\left(\mathrm{~d},{ }^{2} J_{\mathrm{C}-\mathrm{F}}=40.4 \mathrm{~Hz}\right), 135.0,134.5(\mathrm{~d}$, $\left.{ }^{2} J_{\mathrm{C}-\mathrm{F}}=10.9 \mathrm{~Hz}\right), 131.7,131.6\left(\mathrm{~d},{ }^{2} J_{\mathrm{C}-\mathrm{F}}=14.5 \mathrm{~Hz}\right), 130.7(\mathrm{q}$, $\left.{ }^{2} J_{\mathrm{C}-\mathrm{F}}=31.0 \mathrm{~Hz}\right), 129.8,129.2,127.9\left(\mathrm{~d},{ }^{2} J_{\mathrm{C}-\mathrm{F}}=21.4 \mathrm{~Hz}\right)$, $127.1\left(\mathrm{~d},{ }^{2} J_{\mathrm{C}-\mathrm{F}}=38.4 \mathrm{~Hz}\right), 125.0\left(\mathrm{~d},{ }^{3} J_{\mathrm{C}-\mathrm{F}}=4.2 \mathrm{~Hz}\right), 123.3$, $122.0\left(\mathrm{q},{ }^{1} J_{\mathrm{C}-\mathrm{F}}=271.6 \mathrm{~Hz}\right), 120.6\left(\mathrm{~d},{ }^{2} J_{\mathrm{C}-\mathrm{F}}=11.4 \mathrm{~Hz}\right)$, $120.0\left(\mathrm{qd},{ }^{1} J_{\mathrm{C}-\mathrm{F}}=283.6,{ }^{2} J_{\mathrm{C}-\mathrm{F}}=25.9 \mathrm{~Hz}\right), 94.3 \sim 87.0(\mathrm{~m})$, 54.2, 15.2, 9.7, 3.5. HRMS (ESI) cacld for $\mathrm{C}_{27} \mathrm{H}_{17} \mathrm{BrF}_{11^{-}}$ $\mathrm{N}_{2} \mathrm{O}_{2} \mathrm{~S}[\mathrm{M}-\mathrm{H}]^{-}$721.0018, found 721.0007 .

$N$-[2-溴-4-(1,1,1,2,3,3,3-七氟丙-2-基)-6-(三氟甲基) 苯基]-3-[ $N$-(环丙甲基)-3,4-二氯异噻唑-5-甲酰胺基]-2氟苯甲酰胺(6b): 黄色固体, 产率 $35.20 \%$, m.p. 81 $82{ }^{\circ} \mathrm{C} ;{ }^{1} \mathrm{H}$ NMR (400 MHz, DMSO- $\left.d_{6}\right) \delta: 10.70(\mathrm{~s}, 1 \mathrm{H})$, $8.43(\mathrm{~s}, 1 \mathrm{H}), 7.97(\mathrm{~s}, 1 \mathrm{H}), 7.85(\mathrm{t}, J=7.1 \mathrm{~Hz}, 1 \mathrm{H}), 7.70(\mathrm{~m}$, 1H), 7.42 (t, $J=7.6 \mathrm{~Hz}, 1 \mathrm{H}), 3.81(\mathrm{~s}, 1 \mathrm{H}), 3.75 \sim 3.65(\mathrm{~m}$, 1H), $1.09 \sim 0.95(\mathrm{~m}, 1 \mathrm{H}), 0.44$ (d, $J=7.2 \mathrm{~Hz}, 2 \mathrm{H}), 0.15$ (s, $2 \mathrm{H}) ;{ }^{13} \mathrm{C} \mathrm{NMR}\left(100 \mathrm{MHz}, \mathrm{CDCl}_{3}\right) \delta: 160.3,159.6,156.6$ $\left(\mathrm{d},{ }^{1} J_{\mathrm{C}-\mathrm{F}}=248.6 \mathrm{~Hz}\right), 154.0,148.5,136.4,134.7,134.5(\mathrm{~d}$, $\left.{ }^{2} J_{\mathrm{C}-\mathrm{F}}=11.0 \mathrm{~Hz}\right), 133.4,130.8\left(\mathrm{q},{ }^{2} J_{\mathrm{C}-\mathrm{F}}=29.5 \mathrm{~Hz}\right), 129.1(\mathrm{~d}$, $\left.{ }^{2} J_{\mathrm{C}-\mathrm{F}}=15.2 \mathrm{~Hz}\right), 128.2\left(\mathrm{~d},{ }^{2} J_{\mathrm{C}-\mathrm{F}}=21.4 \mathrm{~Hz}\right), 127.3,125.6(\mathrm{~d}$, $\left.{ }^{3} J_{\mathrm{C}-\mathrm{F}}=4.3 \mathrm{~Hz}\right), 123.3,122.0\left(\mathrm{q},{ }^{1} J_{\mathrm{C}-\mathrm{F}}=271.8 \mathrm{~Hz}\right), 121.3$, $121.0\left(\mathrm{~d},{ }^{2} J_{\mathrm{C}-\mathrm{F}}=11.1 \mathrm{~Hz}\right), 120.0\left(\mathrm{qd},{ }^{1} J_{\mathrm{C}-\mathrm{F}}=283.1,{ }^{2} J_{\mathrm{C}-\mathrm{F}}=\right.$ $26.5 \mathrm{~Hz}), 97.5 \sim 84.7(\mathrm{~m}), 54.1,9.3,3.7\left(\mathrm{~d},{ }^{2} J_{\mathrm{C}-\mathrm{F}}=39.3\right.$ $\mathrm{Hz}$ ). HRMS (ESI) cacld for $\mathrm{C}_{25} \mathrm{H}_{12} \mathrm{BrCl}_{2} \mathrm{~F}_{11} \mathrm{~N}_{3} \mathrm{O}_{2} \mathrm{~S}$ [M$\mathrm{H}]^{-}$775.9035, found 775.9031 .

$N$-[2-溴-4-(1,1,1,2,3,3,3-七氟丙-2-基)-6-(三氟甲基) 苯基]-3-[ $\mathrm{N}$-(环丙甲基)-3-(二氟甲基)-1-甲基- $1 \mathrm{H}$-吡唑-4甲酰胺基]-2-氟苯甲酰胺(6c)：黄色固体，产率 $25.20 \%$, m.p. 80 $81{ }^{\circ} \mathrm{C} ;{ }^{1} \mathrm{H}$ NMR (400 MHz, DMSO- $d_{6}$ ) $\delta: 10.65$ $(\mathrm{s}, 1 \mathrm{H}), 8.43(\mathrm{~d}, J=2.0 \mathrm{~Hz}, 1 \mathrm{H}), 7.97(\mathrm{~d}, J=2.1 \mathrm{~Hz}, 1 \mathrm{H})$, $7.83 \sim 7.60$ (m, 2H), 7.44 (t, $J=7.8 \mathrm{~Hz}, 1 \mathrm{H}), 7.12$ (td, $J=$ $54.1,10.7 \mathrm{~Hz}, 2 \mathrm{H}), 3.89 \sim 3.54(\mathrm{~m}, 5 \mathrm{H}), 1.06 \sim 0.99(\mathrm{~m}$, $1 \mathrm{H}), 0.41$ (d, $J=7.1 \mathrm{~Hz}, 2 \mathrm{H}), 0.07$ (s, 2H); ${ }^{13} \mathrm{C}$ NMR (100
$\left.\mathrm{MHz}, \mathrm{CDCl}_{3}\right) \delta: 162.6\left(\mathrm{~d},{ }^{2} J_{\mathrm{C}-\mathrm{F}}=15.1 \mathrm{~Hz}\right), 160.8,156.6(\mathrm{~d}$, $\left.{ }^{1} J_{\mathrm{C}-\mathrm{F}}=248.8 \mathrm{~Hz}\right), 139.3,136.6,135.0,134.5\left(\mathrm{~d},{ }^{2} J_{\mathrm{C}-\mathrm{F}}=\right.$ $10.8 \mathrm{~Hz}), 132.1\left(\mathrm{~d},{ }^{2} J_{\mathrm{C}-\mathrm{F}}=39.6 \mathrm{~Hz}\right), 130.7\left(\mathrm{q},{ }^{2} J_{\mathrm{C}-\mathrm{F}}=29.9\right.$ $\mathrm{Hz}), 130.5,128.1\left(\mathrm{~d},{ }^{2} J_{\mathrm{C}-\mathrm{F}}=21.1 \mathrm{~Hz}\right), 127.3\left(\mathrm{~d},{ }^{3} J_{\mathrm{C}-\mathrm{F}}=2.3\right.$ $\mathrm{Hz}), 125.5\left(\mathrm{~d},{ }^{3} J_{\mathrm{C}-\mathrm{F}}=4.2 \mathrm{~Hz}\right), 123.3,122.0\left(\mathrm{q},{ }^{1} J_{\mathrm{C}-\mathrm{F}}=271.5\right.$ $\mathrm{Hz}), 121.5\left(\mathrm{~d},{ }^{2} J_{\mathrm{C}-\mathrm{F}}=12.2 \mathrm{~Hz}\right), 121.2\left(\mathrm{~d},{ }^{2} J_{\mathrm{C}-\mathrm{F}}=11.2 \mathrm{~Hz}\right)$, $120.0\left(\mathrm{qd},{ }^{1} J_{\mathrm{C}-\mathrm{F}}=258.5,{ }^{2} J_{\mathrm{C}-\mathrm{F}}=27.3 \mathrm{~Hz}\right), 115.2,109.8(\mathrm{t}$, $\left.{ }^{1} J_{\mathrm{C}-\mathrm{F}}=234.5 \mathrm{~Hz}\right), 100.5 \sim 85.1(\mathrm{~m}), 53.6(\mathrm{~d}, J=38.7 \mathrm{~Hz})$, 39.4 (d, $J=43.9 \mathrm{~Hz}), 39.4,9.4,3.3\left(\mathrm{~d},{ }^{2} J_{\mathrm{C}-\mathrm{F}}=39.4 \mathrm{~Hz}\right)$. HRMS (ESI) cacld for $\mathrm{C}_{27} \mathrm{H}_{17} \mathrm{BrF}_{13} \mathrm{~N}_{4} \mathrm{O}_{2} \quad[\mathrm{M}-\mathrm{H}]$ 755.0327, found 755.0316.

$N$-[2-溴-4-(1,1,1,2,3,3,3-七氟丙-2-基)-6-(三氟甲基) 苯基]-3-[ $N$-(环丙甲基)苯甲酰胺基]-2-氟苯甲酰胺(6d): 白色固体, 产率 $27.84 \%$, m.p. $172 \sim 173{ }^{\circ} \mathrm{C} ;{ }^{1} \mathrm{H}$ NMR (400 MHz, DMSO- $\left.d_{6}\right) \delta: 10.60(\mathrm{~s}, 1 \mathrm{H}), 8.42(\mathrm{~s}, 1 \mathrm{H}), 7.95$ (s, 1H), 7.61 (d, $J=22.2 \mathrm{~Hz}, 2 \mathrm{H}), 7.31$ (brs, 6H), 3.70 (d, $J=20.0 \mathrm{~Hz}, 2 \mathrm{H}), 1.03$ (brs, $1 \mathrm{H}), 0.41(\mathrm{~d}, J=7.5 \mathrm{~Hz}, 2 \mathrm{H})$, 0.09 (s, 2H); ${ }^{13} \mathrm{C}$ NMR (100 MHz, DMSO- $\left.d_{6}\right) \delta: 170.0$, $162.6,154.8\left(\mathrm{~d},{ }^{1} J_{\mathrm{C}-\mathrm{F}}=253.6 \mathrm{~Hz}\right), 138.7,135.8,134.0(\mathrm{~d}$, $\left.{ }^{2} J_{\mathrm{C}-\mathrm{F}}=10.5 \mathrm{~Hz}\right), 133.7,131.8 \sim 131.6(\mathrm{~m}), 131.0(\mathrm{q}$, $\left.{ }^{2} J_{\mathrm{C}-\mathrm{F}}=30.3 \mathrm{~Hz}\right), 129.7,128.7\left(\mathrm{~d},{ }^{3} J_{\mathrm{C}-\mathrm{F}}=5.5 \mathrm{~Hz}\right), 127.9$, $127.3,126.3\left(\mathrm{~d},{ }^{2} J_{\mathrm{C}-\mathrm{F}}=21.3 \mathrm{~Hz}\right), 124.6\left(\mathrm{~d},{ }^{3} J_{\mathrm{C}-\mathrm{F}}=4.4 \mathrm{~Hz}\right)$, $123.6\left(\mathrm{~d},{ }^{2} J_{\mathrm{C}-\mathrm{F}}=13.3 \mathrm{~Hz}\right), 122.7,122.2\left(\mathrm{q},{ }^{1} J_{\mathrm{C}-\mathrm{F}}=273.0\right.$ $\mathrm{Hz}), 119.5\left(\mathrm{td},{ }^{1} J_{\mathrm{C}-\mathrm{F}}=286.0 \mathrm{~Hz},{ }^{2} J_{\mathrm{C}-\mathrm{F}}=27.8 \mathrm{~Hz}\right), 90.8(\mathrm{dt}$, $\left.{ }^{1} J_{\mathrm{C}-\mathrm{F}}=203.6 \mathrm{~Hz},{ }^{2} J_{\mathrm{C}-\mathrm{F}}=33.67 \mathrm{~Hz}\right), 53.0,9.4,3.3,3.2$. HRMS (ESI) cacld for $\mathrm{C}_{28} \mathrm{H}_{17} \mathrm{BrF}_{11} \mathrm{~N}_{2} \mathrm{O}_{2}[\mathrm{M}-\mathrm{H}]^{-}$ 701.0298, found 701.0310.

$N$-[2,6-二溴-4-(1,1,1,2,3,3,3-七氟丙-2-基)苯基]3 -[ $N$-(环丙甲基)苯甲酰胺基]-2-氟苯甲酰胺(6e): 白色固 体, 产率 $27.40 \%$, m.p. $76 \sim 77{ }^{\circ} \mathrm{C} ;{ }^{1} \mathrm{H}$ NMR $(400 \mathrm{MHz}$, $\left.\mathrm{CDCl}_{3}\right) \delta: 8.15 \sim 7.85(\mathrm{~m}, 4 \mathrm{H}), 7.63 \sim 7.49(\mathrm{~m}, 1 \mathrm{H})$, $7.48 \sim 7.15(\mathrm{~m}, 6 \mathrm{H}), 3.86(\mathrm{~d}, J=65.5 \mathrm{~Hz}, 2 \mathrm{H}), 1.15$ (brs, $1 \mathrm{H}), 0.57 \sim 0.39(\mathrm{~m}, 2 \mathrm{H}), 0.20(\mathrm{~d}, J=30.6 \mathrm{~Hz}, 2 \mathrm{H}) ;{ }^{13} \mathrm{C}$ NMR (100 MHz, DMSO- $\left.d_{6}\right) \delta: 170.0,161.7,154.8(\mathrm{~d}$, $\left.{ }^{1} J_{\mathrm{C}-\mathrm{F}}=258.2 \mathrm{~Hz}\right), 139.4,135.7,133.7,131.8 \sim 131.2(\mathrm{~m})$, $129.7,129.0,128.9,128.5,127.9,127.8,127.4,126.6(\mathrm{~d}$, $\left.{ }^{2} J_{\mathrm{C}-\mathrm{F}}=21.3 \mathrm{~Hz}\right), 128.8\left(\mathrm{~d},{ }^{3} J_{\mathrm{C}-\mathrm{F}}=1.79 \mathrm{~Hz}\right), 124.6(\mathrm{~d}$, $\left.{ }^{3} J_{\mathrm{C}-\mathrm{F}}=4.2 \mathrm{~Hz}\right), 118.5\left(\mathrm{td},{ }^{1} J_{\mathrm{C}-\mathrm{F}}=285.4 \mathrm{~Hz},{ }^{2} J_{\mathrm{C}-\mathrm{F}}=27.68\right.$ $\mathrm{Hz}), 89.6\left(\mathrm{dt},{ }^{1} J_{\mathrm{C}-\mathrm{F}}=177.8 \mathrm{~Hz},{ }^{2} J_{\mathrm{C}-\mathrm{F}}=28.7 \mathrm{~Hz}\right), 53.0,9.4$, 3.4, 3.2. HRMS (ESI) cacld for $\mathrm{C}_{27} \mathrm{H}_{17} \mathrm{Br}_{2} \mathrm{~F}_{8} \mathrm{~N}_{2} \mathrm{O}_{2}$ [M$\mathrm{H}]^{-}$710.9529, found 710.9545 .

$N$-[2- 甲基-4-(1,1,1,2,3,3,3-七氟丙-2-基)-6-溴苯 基]-3-[ $N$-(环丙甲基)苯甲酰胺基]-2-氟苯甲酰胺(6f): 黄 色固体, 产率 26.99\%, m.p. 87 $88{ }^{\circ} \mathrm{C} ;{ }^{1} \mathrm{H}$ NMR (400 $\left.\mathrm{MHz}, \mathrm{CDCl}_{3}\right) \delta: 7.95(\mathrm{~d}, J=37.9 \mathrm{~Hz}, 2 \mathrm{H}), 7.72(\mathrm{~s}, 1 \mathrm{H})$, 
$7.55 \sim 7.49(\mathrm{~m}, 1 \mathrm{H}), 7.47(\mathrm{~s}, 1 \mathrm{H}), 7.35 \sim 7.20(\mathrm{~m}, 6 \mathrm{H})$, $3.83(\mathrm{~d}, J=55.0 \mathrm{~Hz}, 2 \mathrm{H}), 2.36$ (s, 3H), 1.12 (brs, $1 \mathrm{H}$ ), $0.55 \sim 0.41(\mathrm{~m}, 2 \mathrm{H}), 0.18(\mathrm{~d}, J=25.2 \mathrm{~Hz}, 2 \mathrm{H}) ;{ }^{13} \mathrm{C}$ NMR $\left(100 \mathrm{MHz}, \mathrm{DMSO}-d_{6}\right) \delta: 170.0,161.8,154.6\left(\mathrm{~d},{ }^{1} J_{\mathrm{C}-\mathrm{F}}=\right.$ $251.1 \mathrm{~Hz}), 140.3,138.6,135.8,133.4,131.8 \sim 131.2(\mathrm{~m})$, $129.7,128.8,127.9,127.4,126.8\left(\mathrm{~d},{ }^{2} J_{\mathrm{C}-\mathrm{F}}=11.8 \mathrm{~Hz}\right)$, $126.5\left(\mathrm{~d},{ }^{2} J_{\mathrm{C}-\mathrm{F}}=9.5 \mathrm{~Hz}\right), 125.1\left(\mathrm{~d},{ }^{2} J_{\mathrm{C}-\mathrm{F}}=20.7 \mathrm{~Hz}\right), 124.6$ $\left(\mathrm{d},{ }^{3} J_{\mathrm{C}-\mathrm{F}}=4.3 \mathrm{~Hz}\right), 124.3,124.1,118.6\left(\mathrm{td},{ }^{1} J_{\mathrm{C}-\mathrm{F}}=285 . \mathrm{Hz}\right.$, $\left.{ }^{2} J_{\mathrm{C}-\mathrm{F}}=28.0 \mathrm{~Hz}\right), 90.4\left(\mathrm{dt},{ }^{1} J_{\mathrm{C}-\mathrm{F}}=201.8 \mathrm{~Hz},{ }^{2} J_{\mathrm{C}-\mathrm{F}}=33.8\right.$ $\mathrm{Hz}$ ), 53.0, 18.6, 9.4, 3.4, 3.1. HRMS (ESI) cacld for $\mathrm{C}_{28} \mathrm{H}_{20} \mathrm{BrF}_{8} \mathrm{~N}_{2} \mathrm{O}_{2}[\mathrm{M}-\mathrm{H}]^{-}$647.0580, found 647.0597.

$N$-[2,6-二甲基-4-(1,1,1,2,3,3,3- 七氟丙-2- 基) 苯 基]-3-[ $N$-(环丙甲基)苯甲酰胺基]-2-氟苯甲酰胺(6g): 白 色固体, 产率 40.13\%, m.p. 82 83 ${ }^{\circ} \mathrm{C} ;{ }^{1} \mathrm{H}$ NMR $(400$ $\left.\mathrm{MHz}, \mathrm{CDCl}_{3}\right) \delta: 8.07 \sim 7.91(\mathrm{~m}, 1 \mathrm{H}), 7.51(\mathrm{t}, J=7.7 \mathrm{~Hz}$, 2H), $7.42 \sim 7.15(\mathrm{~m}, 8 \mathrm{H}), 3.83(\mathrm{~d}, J=58.7 \mathrm{~Hz}, 2 \mathrm{H}), 2.27$ (s, $6 \mathrm{H}), 1.12(\mathrm{~s}, 1 \mathrm{H}), 0.69 \sim 0.41(\mathrm{~m}, 2 \mathrm{H}), 0.36 \sim 0.06(\mathrm{~m}$, $2 \mathrm{H}) ;{ }^{13} \mathrm{C}$ NMR (100 MHz, Chloroform- $d$ ) $\delta: 171.0,160.7$, $155.8\left(\mathrm{~d},{ }^{1} J_{\mathrm{C}-\mathrm{F}}=249.7 \mathrm{~Hz}\right), 136.3\left(\mathrm{~d},{ }^{2} J_{\mathrm{C}-\mathrm{F}}=10.7 \mathrm{~Hz}\right)$, 135.6, 133.7, 131.3, 130.0, 127.8, 125.7, $125.5\left(\mathrm{~d},{ }^{2} J_{\mathrm{C}-\mathrm{F}}=\right.$ $10.4 \mathrm{~Hz}), 125.1\left(\mathrm{~d},{ }^{3} J_{\mathrm{C}-\mathrm{F}}=4.3 \mathrm{~Hz}\right), 120.5\left(\mathrm{qd},{ }^{1} J_{\mathrm{C}-\mathrm{F}}=284.0\right.$, $\left.{ }^{2} J_{\mathrm{C}-\mathrm{F}}=27.9 \mathrm{~Hz}\right), 104.4 \sim 86.0(\mathrm{~m}), 54.0,18.8,9.7,3.6$. HRMS (ESI) cacld for $\mathrm{C}_{29} \mathrm{H}_{23} \mathrm{~F}_{8} \mathrm{~N}_{2} \mathrm{O}_{2}[\mathrm{M}-\mathrm{H}]^{-}$583.1632, found 583.1622 .

$N$-[2,6-二乙基-4-(1,1,1,2,3,3,3-七氟丙-2-基)苯基]3-[ $N$-(环丙甲基)苯甲酰胺基]-2-氟苯甲酰胺 $(\mathbf{6 h})$ : 白色 固体, 产率 50.13\%, m.p. $68 \sim 69{ }^{\circ} \mathrm{C} ;{ }^{1} \mathrm{H}$ NMR $(400 \mathrm{MHz}$, $\left.\mathrm{CDCl}_{3}\right) \delta: 7.99(\mathrm{t}, J=7.5 \mathrm{~Hz}, 1 \mathrm{H}), 7.53(\mathrm{t}, J=7.9 \mathrm{~Hz}, 2 \mathrm{H})$, $7.45 \sim 7.24(\mathrm{~m}, 6 \mathrm{H}), 7.19(\mathrm{~d}, J=10.2 \mathrm{~Hz}, 2 \mathrm{H}), 3.83$ (d, $J=$ $89.7 \mathrm{~Hz}, 2 \mathrm{H}), 2.59$ (q, $J=7.7 \mathrm{~Hz}, 4 \mathrm{H}), 1.35 \sim 1.24(\mathrm{~m}$, $1 \mathrm{H}), 1.17$ (t, $J=7.6 \mathrm{~Hz}, 6 \mathrm{H}), 0.49$ (t, $J=7.2 \mathrm{~Hz}, 2 \mathrm{H}), 0.19$ (d, $J=36.2 \mathrm{~Hz}, 2 \mathrm{H}) ;{ }^{13} \mathrm{C}$ NMR $\left(100 \mathrm{MHz}, \mathrm{CDCl}_{3}\right) \delta$ : $170.9,161.4,142.3,135.6,135.0,133.7,131.4\left(\mathrm{~d},{ }^{3} J_{\mathrm{C}-\mathrm{F}}=\right.$ $2.2 \mathrm{~Hz}), 129.9,127.8,126.4\left(\mathrm{~d},{ }^{2} J_{\mathrm{C}-\mathrm{F}}=20.1 \mathrm{~Hz}\right), 125.2(\mathrm{~d}$, $\left.{ }^{3} J_{\mathrm{C}-\mathrm{F}}=4.2 \mathrm{~Hz}\right), 123.7\left(\mathrm{~d},{ }^{2} J_{\mathrm{C}-\mathrm{F}}=10.2 \mathrm{~Hz}\right), 120.5(\mathrm{dd}$, $\left.{ }^{1} J_{\mathrm{C}-\mathrm{F}}=284.0,{ }^{2} J_{\mathrm{C}-\mathrm{F}}=27.8 \mathrm{~Hz}\right), 95.6 \sim 88.5(\mathrm{~m}), 54.0,25.1$, 14.0, 9.7, 3.6. HRMS (ESI) cacld for $\mathrm{C}_{31} \mathrm{H}_{27} \mathrm{~F}_{8} \mathrm{~N}_{2} \mathrm{O}_{2}$ [M$\mathrm{H}]^{-}$611.1945, found 611.1930.

$N$-[2-溴-4,6-二(三氟甲基)苯基]-3-[ $N$-(环丙甲基)苯 甲酰胺基]-2-氟苯甲酰胺(6i): 白色固体, 产率 48.97\%, m.p. 83 84 ${ }^{\circ} \mathrm{C} ;{ }^{1} \mathrm{H}$ NMR (400 MHz, $\mathrm{CDCl}_{3}$ ) $\delta: 8.15$ (s, 1H), $8.06 \sim 7.96(\mathrm{~m}, 2 \mathrm{H}), 7.94(\mathrm{~s}, 1 \mathrm{H}), 7.52(\mathrm{t}, J=6.7 \mathrm{~Hz}$, 1H), $7.38 \sim 7.15(\mathrm{~m}, 6 \mathrm{H}), 3.82(\mathrm{~d}, J=61.3 \mathrm{~Hz}, 2 \mathrm{H}), 1.11$ (s, $1 \mathrm{H}), 0.49$ (d, $J=6.8 \mathrm{~Hz}, 2 \mathrm{H}), 0.17$ (d, $J=26.6 \mathrm{~Hz}, 2 \mathrm{H}$ ); ${ }^{13} \mathrm{C}$ NMR (100 MHz, DMSO- $\left.d_{6}\right) \delta: 170.0,162.5,154.8(\mathrm{~d}$, $\left.{ }^{1} J_{\mathrm{C}-\mathrm{F}}=255.7 \mathrm{~Hz}\right), 138.8,135.7,134.2\left(\mathrm{~d},{ }^{3} J_{\mathrm{C}-\mathrm{F}}=3.9 \mathrm{~Hz}\right)$, $133.6,131.9 \sim 131.1(\mathrm{~m}), 130.6\left(\mathrm{q},{ }^{2} J_{\mathrm{C}-\mathrm{F}}=30.64 \mathrm{~Hz}\right), 130.0$ $\left(\mathrm{q},{ }^{2} J_{\mathrm{C}-\mathrm{F}}=33.66 \mathrm{~Hz}\right), 129.7,128.8,127.8,127.4,124.6(\mathrm{~d}$, $\left.{ }^{3} J_{\mathrm{C}-\mathrm{F}}=4.5 \mathrm{~Hz}\right), 123.7\left(\mathrm{~d},{ }^{2} J_{\mathrm{C}-\mathrm{F}}=13.5 \mathrm{~Hz}\right), 123.3,122.3(\mathrm{q}$, $\left.{ }^{1} J_{\mathrm{C}-\mathrm{F}}=271.69 \mathrm{~Hz}\right), 121.9\left(\mathrm{q},{ }^{1} J_{\mathrm{C}-\mathrm{F}}=272.72 \mathrm{~Hz}\right), 53.0,9.4$, 3.3, 3.1. HRMS (ESI) cacld for $\mathrm{C}_{26} \mathrm{H}_{17} \mathrm{BrF}_{7} \mathrm{~N}_{2} \mathrm{O}_{2}[\mathrm{M}-\mathrm{H}]^{-}$ 601.0362, found 601.0374 .

$N$-[2,6-二溴-4-三氟甲氧基苯基]-3-[ $N$-(环丙甲基)苯 甲酰胺基]-2-氟苯甲酰胺 $(\mathbf{6 j})$ : 白色固体，产率 38.23\%, m.p. $98 \sim 99{ }^{\circ} \mathrm{C} ;{ }^{1} \mathrm{H}$ NMR (400 MHz, DMSO- $\left.d_{6}\right) \delta: 10.36$ (s, 1H), $7.94(\mathrm{~s}, 2 \mathrm{H}), 7.60(\mathrm{~s}, 2 \mathrm{H}), 7.42 \sim 7.18(\mathrm{~m}, 6 \mathrm{H})$, $3.69(\mathrm{~s}, 1 \mathrm{H}), 3.62(\mathrm{~s}, 1 \mathrm{H}), 1.1 \sim 0.95(\mathrm{~m}, 1 \mathrm{H}), 0.48 \sim 0.35$ $(\mathrm{m}, 2 \mathrm{H}), 0.18 \sim 0.05(\mathrm{~m}, 2 \mathrm{H}) ;{ }^{13} \mathrm{C}$ NMR $(100 \mathrm{MHz}$, $\left.\mathrm{CDCl}_{3}\right) \delta: 171.0,160.6,156.1\left(\mathrm{~d},{ }^{1} J_{\mathrm{C}-\mathrm{F}}=247.4 \mathrm{~Hz}\right), 148.1$, $138.5,135.5,134.3,133.5,132.3,131.5,130.0,127.9$, $125.0\left(\mathrm{~d},{ }^{3} J_{\mathrm{C}-\mathrm{F}}=4.3 \mathrm{~Hz}\right), 124.8,124.1,124.0\left(\mathrm{~d},{ }^{3} J_{\mathrm{C}-\mathrm{F}}=6.8\right.$ $\mathrm{Hz}), 121.0,120.1\left(\mathrm{q},{ }^{1} J_{\mathrm{C}-\mathrm{F}}=257.3 \mathrm{~Hz}\right), 119.0,53.9,30.2$, 9.7, 3.6. HRMS (ESI) cacld for $\mathrm{C}_{25} \mathrm{H}_{17} \mathrm{Br}_{2} \mathrm{~F}_{4} \mathrm{~N}_{2} \mathrm{O}_{3}$ [M$\mathrm{H}]^{-}$626.9542, found 626.9536 .

$N$-[2-溴-4-(1,1,1,2,3,3,3-七氟丙-2-基)-6-(三氟甲基) 苯基]-3-[ $N$-(环丙甲基)肉桂酰胺]-2-氟苯甲酰胺 $(\mathbf{6 k}$ ): 白 色固体, 产率 $18.23 \%$, m.p. $121 \sim 122{ }^{\circ} \mathrm{C} ;{ }^{1} \mathrm{H}$ NMR (400 MHz, DMSO- $\left.d_{6}\right) \delta: 10.78(\mathrm{~s}, 1 \mathrm{H}), 8.43(\mathrm{~s}, 1 \mathrm{H}), 7.97(\mathrm{~s}$, $1 \mathrm{H}), 7.79(\mathrm{t}, J=6.1 \mathrm{~Hz}, 1 \mathrm{H}), 7.75 \sim 7.67(\mathrm{~m}, 1 \mathrm{H}), 7.64(\mathrm{~s}$, $1 \mathrm{H}), 7.60(\mathrm{~s}, 1 \mathrm{H}), 7.51(\mathrm{t}, J=7.7 \mathrm{~Hz}, 1 \mathrm{H}), 7.48 \sim 7.38(\mathrm{~m}$, 2H), $7.38 \sim 7.30(\mathrm{~m}, 3 \mathrm{H}), 6.35(\mathrm{~d}, J=15.4 \mathrm{~Hz}, 1 \mathrm{H}), 3.79$ (s, 1H), 3.54 (s, 1H), 0.97 (s, 1H), 0.41 (d, $J=6.8 \mathrm{~Hz}, 2 \mathrm{H})$, $0.11(\mathrm{~s}, 2 \mathrm{H}) ;{ }^{13} \mathrm{C}$ NMR $\left(100 \mathrm{MHz}, \mathrm{CDCl}_{3}\right) \delta: 169.8,165.7$, $160.8\left(\mathrm{~d},{ }^{3} J_{\mathrm{C}-\mathrm{F}}=3.3 \mathrm{~Hz}\right), 156.6\left(\mathrm{~d},{ }^{1} J_{\mathrm{C}-\mathrm{F}}=249.1 \mathrm{~Hz}\right), 146.1$, $143.4,136.5,135.3,134.5,134.3\left(\mathrm{~d},{ }^{2} J_{\mathrm{C}-\mathrm{F}}=10.6 \mathrm{~Hz}\right)$, $132.1,130.7\left(\mathrm{q},{ }^{2} J_{\mathrm{C}-\mathrm{F}}=30.4\right), 130.5\left(\mathrm{~d},{ }^{2} J_{\mathrm{C}-\mathrm{F}}=15.1 \mathrm{~Hz}\right)$, $130.3\left(\mathrm{~d},{ }^{2} J_{\mathrm{C}-\mathrm{F}}=12.4 \mathrm{~Hz}\right), 129.7,128.6\left(\mathrm{~d},{ }^{2} J_{\mathrm{C}-\mathrm{F}}=14.5 \mathrm{~Hz}\right)$, $128.0\left(\mathrm{~d},{ }^{3} J_{\mathrm{C}-\mathrm{F}}=8.5 \mathrm{~Hz}\right), 127.4\left(\mathrm{~d},{ }^{2} J_{\mathrm{C}-\mathrm{F}}=51.4 \mathrm{~Hz}\right), 125.3$ $\left(\mathrm{d},{ }^{3} J_{\mathrm{C}-\mathrm{F}} J=4.2 \mathrm{~Hz}\right), 123.1,122.0\left(\mathrm{q},{ }^{1} J_{\mathrm{C}-\mathrm{F}}=271.6 \mathrm{~Hz}\right)$, $120.9\left(\mathrm{~d},{ }^{2} J_{\mathrm{C}-\mathrm{F}}=11.4 \mathrm{~Hz}\right), 120.0\left(\mathrm{qd},{ }^{1} J_{\mathrm{C}-\mathrm{F}}=284.4,{ }^{2} J_{\mathrm{C}-\mathrm{F}}=\right.$ $27.2 \mathrm{~Hz}), 117.1\left(\mathrm{~d},{ }^{2} J_{\mathrm{C}-\mathrm{F}}=15.8 \mathrm{~Hz}\right), 97.3 \sim 83.6(\mathrm{~m}), 53.0$, 9.4, $3.3\left(\mathrm{~d},{ }^{2} J_{\mathrm{C}-\mathrm{F}}=37.4 \mathrm{~Hz}\right)$. HRMS (ESI) cacld for $\mathrm{C}_{30} \mathrm{H}_{19} \mathrm{BrF}_{11} \mathrm{~N}_{2} \mathrm{O}_{2}[\mathrm{M}-\mathrm{H}]^{-}$727.0454, found 727.0443.

\section{3 生物活性测试}

试虫小菜蛾 Plutella xylostella 和二化螟 Chilo suppressalis 由上海晓明检测技术服务有限公司提供, 为室 内连续化饲养 3 龄幼虫. 称取 $10 \mathrm{mg}$ 目标化合物, 用 1 $\mathrm{mL} N, N$-二甲基甲酰胺(DMF)溶解，配制成 $10 \mathrm{~g} / \mathrm{L}$ 母液. 用 $0.05 \%$ 的吐温水稀释, 配制成所需浓度待测液.

小菜蛾杀虫活性测试采用浸叶碟饲喂法: 将甘蓝叶 
碟浸入药液中 $10 \mathrm{~s}$, 晾干后置于培养血中, 每皿 4 碟, 培 养血内放滤纸保湿. 每皿接小菜蛾试虫 10 头, 3 次重复. 置于光照培养箱内, 温度 $25^{\circ} \mathrm{C}$, 光照 $14 \mathrm{~h}$, 黑暗 $10 \mathrm{~h}$ 培养. 药后 $3 \mathrm{~d}$ 调查死活虫数, 并进行统计分析.

二化螟杀虫活性测试采用稻茎浸渍法: 温室内用直 径为 $9 \mathrm{~cm}$ 、高 $10 \mathrm{~cm}$ 的塑料盆钵培养水稻, 待水稻长至 株高约 $25 \mathrm{~cm}$ 时, 选择健壮的、长势一致的水稻幼苗, 剪 取地上部分, 去叶, 保留稻茎, 约 $8 \mathrm{~cm}$ 长, 备用. 将药 液倒入培养血中(药液量约 $40 \mathrm{~mL}$ ), 将稻茎浸入药液. 浸渍 $10 \mathrm{~s}$ 后取出, 放置于阴凉处晾干. 玻璃指形管底部 放保湿棉球, 每管放入 5 根处理后稻茎, 接三龄二化蚯 幼虫 10 头, 每处理重复 3 次, 用棉黑布封管口，橡皮筋 扎紧, 置于光照培养箱, 温度 $28{ }^{\circ} \mathrm{C}$, 黑暗培养. 药后 3 天调查二化蚯活虫数, 药后 $3 \mathrm{~d}$ 同时调查总虫数, 计算 各药剂处理的死亡率.

辅助材料(Supporting Information) 中间体 8, 10a $10 \mathrm{k}, 11 \mathrm{a} \sim 11 \mathrm{k}$ 及目标产物 $6 \mathbf{a} \sim \mathbf{6 k}$ 的 ${ }^{1} \mathrm{H} \mathrm{NMR}$ 和 ${ }^{13} \mathrm{C}$ NMR 谱图. 这些材料可以免费从本刊网站(http://siocjournal.cn/)上下载.

\section{References}

[1] Zhou, S.; Gu, Y. C.; Liu, M.; Wu, C. C.; Zhou, S.; Zhao, Y.; Jia, Z. H.; Wang, B. L.; Xiong, L. X.; Yang, N.; Li, Z. M. J. Agric. Food Chem. 2014, 62, 11054.

[2] Zhou, S.; Yan, T.; Li, Y. X.; Jia, Z. H.; Wang, B. L.; Zhao, Y.; Qiao, Y. Y.; Xiong, L. X.; Li, Y. Q.; Li, Z. M. Org. Biomol. Chem. 2014, 12,6643 .

[3] Li, Y. H.; Zhu, H. J; Chen, K.; Liu, R.; Khallaf, A; Zhang, X. J; Ni, J. P. Org. Biomol. Chem. 2013, 11, 3979.

[4] Meng, X. D.; Zhou, S.; Xie, Y. T.; Zhao, Y.; Xiong, L. X.; Zhou, S.; Li, Z. M. Chin. J. Org. Chem. 2017, 37, 908 (in Chinese). (孟祥德, 周沙, 解永涛, 赵毓, 熊丽霞, 周莎, 李正名, 有机化 学, 2017, 37, 908.)

[5] Li, Y.; Li, M.; Chai, B. S; Liu, C. L. Agrochemicals 2005, 45, 697 (in Chinese). (李洋, 李丞, 柴宝山, 刘长令, 农药, 2005, 45, 697.)

[6] Qu, J. J.; Zhu, X. K; Wang, L.; Xu, C.; Liu, F.; Ren, L.; Xu, X. B.; Xiong, L. X.; Wang, Y.; Rui, C. H.; Liu, S. D. J. Agric. Food Chem. 2012, 60, 10942.

[7] Zhou, Y. Y.; Wang, B. L; Di, F. J; Xu, C.; Xiong, L. X.; Yang, N.; Li, Y. Q; Li, Y. X.; Li, Z. M. Bioorg. Med. Chem. Lett. 2014, 24, 2295.

[8] Kang, S. H.; Song, B. A; Wu, J; He, M.; Hu, D. Y.; Jin, L. H; Zeng,
S; Xue, W.; Yang, S. Eur. J. Med. Chem. 2014, 67, 14

[9] Guo, L.; Wang, Y.; Zhou, X. G.; Li, Z. Y.; Liu, S. Z.; Liang, P.; Gao, X. W. Pest Manage. Sci. 2014, 70, 1083 .

[10] Guo, L.; Liang, P.; Zhou, X. G.; Gao, X. W. Sci. Rep. 2014, 4, 6924.

[11] Hu, Z. D.; Chen, H. Y.; Li, Z. Y.; Zhang, D. Y.; Yin, F.; Lin, Q. S.; Bao, H. L.; Zhou, X. M.; Feng, X. Guangdong Agric. Sci. 2012, (1), 79 (in Chinese).

(胡珍娣，陈焕瑜，李振宇，张德雍，尹飞，林庆胜，包华理，周 小毛, 冯夏, 广东农业科学, 2012, (1), 79.)

[12] Hu, Z. D.; Feng, X.; Li, Z. Y.; Zhang, D. Y.; Chen, H, Y. Agrochem. Res. Appl. 2010, 14, 25 (in Chinese). (胡珍娣，冯夏，李振宇，张德雍，陈焕瑜，农药研究与应用， 2010, 14,25 .)

[13] Li, X. X.; Liang, P.; Gao, X. W. J. Plant Protect. 2015, 42, 481 (in Chinese).

(李秀霞, 梁沛, 高希武, 植物保护学报, 2015, 42, 481.)

[14] Yang, J. C.; Wu, Q.; Wang, X. L. Agrochemicals 2014, 53, 215 (in Chinese).

(杨吉春，吴峤，王秀丽，农药，2014, 53, 215.)

[15] Nakao, T.; Banba, S. Bioorg. Med. Chem. 2016, 24, 372.

[16] Nakao, T.; Banba, S.; Nomura, M.; Hirase, K. Insect Biochem. Mol. Biol. 2013, 43, 366.

[17] Ozoe, Y.; Kita, T.; Ozoe, F.; Nakao, T.; Sato, K.; Hirase, K. Pestic. Biochem. Physiol. 2013, 107, 285.

[18] Katsuta, H.; Nomura, M.; Wakita, T.; Daido, H.; Kobayashi, Y.; Kawahara, A.; Banba, S. J. Pestic. Sci. 2019, 44, 120

[19] Qi, H. L.; Cui, L.; Wang, Q. Q.; Liu, F.; Bing, C. H. Plant Protect. 2017, 43, 112 (in Chinese).

(齐浩亮, 崔丽, 王芹芹, 刘峰, 芮昌辉, 植物保护, 2017, 43, 112.)

[20] Muriel, B.; Gagnebin, A.; Waser, J. Chem. Sci. 2019, 10, 10716.

[21] Menear, K. A.; Adcock, C.; Boulter, R.; Cockcroft, X.; Copsey, L.; Cranston, A.; Dillon, K. J.; Drzewiecki, J.; Garman, S.; Gomez, S.; Javaid, H.; Kerrigan, F.; Knights, C.; Lau, A.; Vincent, M.; Jr, Moore, S.; Smith, J. C.; Martin, N. M. J. Med. Chem. 2008, 51, 6581.

[22] Chang, F.; Dutta, S.; Becnel, J. J.; Estep, A. S.; Mascal, M. J. Agric. Food Chem. 2014, 62, 476

[23] Liu, A. C.; He, X. L.; Feng, J. L.; Yu, C. H.; Zhang, S. K. Agrochemicals 2015, 54, 790 (in Chinese). (刘安昌，贺晓璐，冯佳丽，余彩虹，张树康，农药，2015，54， 790.)

[24] Dai, H.; Hong, Y.; Ye, L. Y.; Zhong, S. L.; Cao, X. F.; Shi, Y. J.; Li, C. J.; Shi, J.; Shi, L. Chin. J. Org. Chem. 2017, 37, 3006 (in Chinese).

(戴红，洪宇，叶林玉，仲苏林，曹雄飞，石玉军，李春建，石 健，施磊，有机化学, 2017, 37, 3006.)

[25] Zhu, B. C.; Xu, T. M.; Kong, X. L.; Wei, W.; Li, X. N. Agrochemicals 2009, 48, 712 (in Chinese). (朱冰春, 许天明, 孔小林, 韦伟, 李小年, 农药, 2009, 48, 712.)

[26] Zhang, H. M.; Feng, Q; Weng, J. Q.; Kong, X. L.; Wei, Y. C.; Yuan, J.; Xing, J. H. Chin. J. Org. Chem. 2013, 33, 32 (in Chinese). (张红敏, 冯启, 翁建全, 孔小林, 魏优昌, 袁静, 邢家华, 有机 化学, 2013, 33, 32.) 